\title{
Encouraging Speech and Vocalization in Children with Autistic Spectrum Disorder
}

\author{
Joshua Hailpern \\ Department of Computer Science \\ jhailpe2@cs.uiuc.edu \\ University of Illinois at Urbana-Champaign \\ Urbana, IL 61801 USA \\ Laura DeThorne \\ Department of Speech and Hearing Science \\ lauras@uiuc.edu \\ University of Illinois at Urbana-Champaign \\ Urbana, IL 61801 USA
}

\begin{abstract}
Technology can improve the life of those with Autistic Spectrum Disorder (ASD). Specifically, some children with ASD are not fortunate enough to acquire the ability to communicate with language on their own. With language being an important method of communication, socialization, and interacting with the world, these children need researchers to develop new solutions to help teach vocalization and speech. Without speech, these children will have difficulty communicating their needs, wants, and emotions, as well as being able to function within society at large. This paper examines the existing HCI research on ASD, as well as proposes a new direction for investigation.
\end{abstract}

\section{Categories and Subject Descriptors}

H 5.2 [Information Interfaces and Presentation]: Screen design, Voice I/O. K4.2 [Social Issues]: Assistive technologies for persons with disabilities

\section{General Terms}

Human Factors

\section{Keywords}

Accessibility, Autism, Children, Speech, Vocalization, Techniques

\section{Introduction}

During the normal development of a child, language is acquired without much explicit effort by parents, practitioners, or the community. However, some children, such as those with Autistic Spectrum Disorder (ASD), are not fortunate enough to acquire this skill on their own. That language is considered "a unique characteristic of human behavior... [that] contributes in a major way to human thought and reasoning"[11], these children need our help to learn to communicate and function in the "real world". Without speech, these children have difficulty expressing their desires, emotions, and communicating on a day-to-day basis. HCI

\author{
Karrie Karahalios \\ Department of Computer Science \\ kkarahal@cs.uiuc.edu \\ University of Illinois at Urbana-Champaign \\ Urbana, IL 61801 USA \\ James Halle \\ Department of Special Education \\ halle@uiuc.edu \\ University of Illinois at Urbana-Champaign \\ Urbana, IL 61801 USA
}

is poised to develop new techniques that use technology to assist practitioners and parents to teach their children with ASD to talk and develop normally. With 1 in 150 children being diagnosed with ASD [4 2007], the need for new solutions is growing.

\section{A Brief Description of Autistic Spectrum Disorder}

ASD is a developmental disability impacting social functioning, such as empathy, basic interaction and communication. It is important to note that autism affects each individual differently. These differences cause a wide range, or spectrum, or conditions ranging from individuals who are "high-functioning" or having only slight delays in communication and social functioning, to those who are "low-functioning" having a greater challenge of interacting. Other notable characteristics of many individuals with autism include "insistence on sameness... Preference to being alone... spinning objects [and] obsessive attachments to objects “ [1].

\section{Communication Treatments}

In the 1960s, Ivar Lovaas began teaching children with autism new behaviors through a technique called "applied behavior analysis", in which a behavior is encouraged or discouraged as it encounters environmental consequences. In short, his technique relies upon using objects, food, and actions as rewards for desired behavior (prompted by a researcher) [11]. Over many trials and sessions, children with autism eventually learn to respond in a predictable fashion by interacting with people in their environment. There are three main drawbacks to this form of treatment:

- It requires many sessions with trained professionals who are in short supply. This can place a financial burden on the family.

- Teaching sessions require intense attention and prolonged contact from a practitioner or parent.

- The child must interact with a human being. One characteristic of ASD is anxious, detached, and "alone" interaction with other individuals $[2,6]$. Thus the interaction with a human being, as the primary mode of teaching, might pose some degree of built-in difficulty for the ASD child.

\section{HCI Research On ASD Treatments}

Existing work by HCI researchers has approached ASD from three primary directions. Work by Abowd and others have explored the benefits of technology to aid the diagnosis process $[5,8,9]$. This research is crucial, because early detection allows children to begin 
treatment earlier, allowing them to catch up faster to their nonautistic peers. Further, this work allows us to better understand how to identify autistic characteristics. Although greatly beneficial, this research does not provide a direct method to enhance the education of children with ASD.

Researchers have also explored the effect that technological environments have had on the process of assisting children with autism to learn how to interact with other human beings [7, 14]. This work uses virtual environments, as well as virtual peers, to create situations in which the children with ASD are comfortable. They are then able to learn person-to-person interactions, without the apprehension of having another person in the room. This work, however, primarily has dealt with "high functioning" children, or those who already know how to speak and have a deficit in social interaction. Therefore, it is hoped that principles learned from this body of literature will have the potential to be applied to research targeting children with ASD who have not yet acquired speech.

The third approach seeks to encourage children with ASD to "play," where playing is mediated through technology $[10,12,13]$. By creating technological methods of interaction (visual displays and physical robots), play and comfortable interactions can be garnered from children with autism. There is a feeling of "safety" by having the main form of interaction occur with non-humans. Further, these devices allow the child, rather than a third party, to be in control of the interactions. This research has much potential. To date, however, it has not focused on encouraging more communication-based activities, such as speech and human-tohuman interaction.

\section{A Proposal for a New Direction in Research}

Through this analysis of existing approaches, we believe there is potential to create a new direction of research, focusing on using technology to encourage meaningful speech in low-functioning children with ASD. Speech would allow these children to express needs, desires and live more normal lives in society. The literature provides strong evidence that interacting with technology often can motivate children with ASD. Further, existing literature shows that real-time visualizations, which act as social mirrors, can influence communication interaction [3]. Therefore, we see the potential of technology to aid teachers in the development of sounds, words, and speech; thereby contributing to what is an exclusively human-to-human interaction. By introducing technology into this form of treatment, we believe we can alleviate a degree of apprehension experienced by the children when interacting with humans, and provide teachers with a new technique to complement and supplement their existing approaches.

Some of the literature has successfully encouraged play by leveraging visualizations that use abstract imagery (shapes that do not directly map to real world objects). Further, research targeting human-human interaction through training on virtual environments shows the potential benefits of training with this technology. We believe that combining aspects of these two approaches could facilitate a new understanding of sound and speech. These results lead us to believe that a similar approach could be used to help children with ASD understand and control their sounds by visualizing their voice. These visualizations could be used as form of reward for making correct sounds, or as a form of feedback to allow them to visually comprehend the sounds they are making.
By constructing these visualizations using hand held devices, the learning experience could also be easily extended to day-to-day life, rather than being limited to an office or a school.

\section{Current Research}

In order to test whether computer generated stimuli (auditory and/or visual) can be constructed to encourage/reinforce sound production in children with ASD, we have begun the first phase of this research project. Our study attempts to uncover the effect of different permutations of auditory and visual feedback on the vocal production of children in our target population. We have constructed approximately a dozen varying types of visualizations, as well as 4 different metaphors for sound feedback.

Children enrolled in this first phase of research attend 6 sessions in which a researcher presents them with approximately 8 different forms of feedback to sound production over about a 40-minute period. Each combination of visual and auditory feedback (trial) is separated by a period of play and relaxation for the child, in order to maintain a positive and stress-free environment. Software, on the systems presenting the feedback, logs critical data points about the child's interaction with the software. With parental consent, sessions are video taped to allow researchers to gather additional data.

From these sessions, research will attempt to determine not only whether these forms of feedback can encourage sound, but also explore other related behaviors of children with ASD; is there a form of feedback (auditory or visual) that is more influential, what type and amount of attention will a child pay to the screen on a computer, are children with ASD visual learners, and are all children with ASD the same (in terms of preferences).

Phase One began in early August 2007. It is expected to be completed by middle to late October 2007. Initial qualitative results appear promising. Qualitatively, many of the children appear to have forms of feedback for which they will generate far greater number (and frequency) of sounds. In addition, many parents have expressed that their children become excited, if not elated when they realize that they are coming to a session. That could indicate that the visual/auditory stimulus is enjoyable to play with and thus could be used as a form of enjoyable education for children with ASD.

One specific child appears to be reacting over and above the others in terms of sound production. Specifically, he appears to be exhibiting signs of turn taking (with the auditory feedback), as well as mimicking many of the sounds generated (as if playing a call and response game). At the completion of his standard 6 sessions, we are planning to conduct an additional 2 sessions with a new visualization (controlled through a wizard of $\mathrm{Oz}$ technique) that requests that the child make specific sounds. Should those sounds be made, visual and auditory reinforcement will be provided. The choice of reinforcement will be based on the qualitative observations of the child's preferences made by researchers. Should the results prove positive, we will have an indication that these systems (with little modification) could be used to teach sound formation to some children with ASD.

For the quantitative analysis, we plan to treat Phase One as a single subject analysis across conditions with auditory and video 
recordings of the following variables: time in chair, percent of intervals with positive emotive state, diversity of phonetic repertoire, frequency of utterances, variation in utterance duration, and exploration of utterance volume. Though we plan to focus primarily on within subject comparisons, we do plan to do some across-subject analysis.

Further studies are planned exploring the effect of visualizations on vocalization frequency, controlling vocalization, instructing children to make specific sounds, and word formation. We also are planning to explore form factors ranging from larger objects to portable hand-held devices (which would allow children to learn outside of an office, in more comfortable environments like the home).

\section{Conclusion}

To date, little or no research has been reported on using technology to teach low functioning children with ASD to learn to vocalize or speak. However, the field of augmentative and alternative communication embraces technology primarily as a medium of communication and not as often as a method of instruction. If we can encourage vocalization at the age of 3 , a pivotal age for children with ASD, this could lead to an increased communicative ability, which makes not only the child's life easier, but also increases their chances of functioning in the world around them. In addition, if the approach we are recommending proves successful for speech, similar methods could be applied to other behaviors or to other disorders with speech impairments.

\section{References}

1. Autism Society of America, ASA. Autism Society of America, 2007.

2. Baskett, C.B. The effect of live interactive video on the communicative behavior in children with autism, University of North Carolina at Chapel Hill, Chapel Hill, 1996.

3. Bergstrom, T. and Karahalios, K., Seeing More: Visualizing Audio Cues. in INTERACT, (Rio de Janerio, Brasil, 2007).

4. Center for Disease Control and Prevention, CDC. Autism Information Center, DD, NCBDDD, CDC, Atlanta, April 25, 2007.

5. Hayes, G.R., Abowd, G.D., et al. Designing Capture Applications to Support the Education of Children with Autism in International Conference on Ubiquitous Computing, (Nottingham, England, 2004).

6. Kanner, L. Autistic Disturbances of Affective Contact. in Kanner, L. ed. Nervous Child 2, V.H. Winston, 1943, 217-250.

7. Kerr, S.J., Neale, H.R. and Cobb, S.V.G. Virtual environments for social skills training: the importance of scaffolding in practice Proceedings of the fifth international ACM conference on Assistive technologies, ACM Press, Edinburgh, Scotland, 2002.

8. Kientz, J.A., Abowd, G.D., et al. Grow and know: understanding recordkeeping needs for tracking the development of young children Proceedings of the SIGCHI conference on Human factors in computing systems, ACM Press, San Jose, California, USA, 2007.

9. Kientz, J.A., Hayes, G.R., Abowd, G.D. and Grinter, R.E. From the war room to the living room: decision support for home-based therapy teams Proceedings of the 2006 20th anniversary conference on Computer supported cooperative work, ACM Press, Banff, Alberta, Canada, 2006.

10. Lehman, J.F. Toward the use of speech and natural language technology in intervention for a language-disordered population Proceedings of the third international ACM conference on Assistive technologies, ACM Press, Marina del Rey, California, United States, 1998.

11. Lovaas, I.I. The Autistic Child. John Wiley \& Sons, Inc, New York, 1977.

12. Michaud, F. and Théberge-Turmel, C. Mobile robotic toys and autism. in Dautenhahn, K. ed. Socially Intelligent Agents - Creating Relationships with Computers and Robots, Springer, 2002, 125-132.

13. Parés, N., Carreras, A., et al. Promotion of creative activity in children with severe autism through visuals in an interactive multisensory environment Proceeding of the 2005 conference on Interaction design and children, ACM Press, Boulder, Colorado, 2005.

14. Tartaro, A. Storytelling with a virtual peer as an intervention for children with autism. SIGACCESS Access. Comput. (84). 42-44. 